\title{
Study of Effect on Performance of DE/BBO on Changing Parametric Values
}

\author{
Ekta $^{1}$ and Mandeep Kaur ${ }^{2}$ \\ ${ }^{1}$ Department of Computer Science, GNDU, RC, Jalandhar \\ ${ }^{2}$ Department of Computer Science, GNDU, RC, Jalandhar \\ ${ }^{1}$ er_ekta@yahoo.com, ${ }^{2}$ mandeep8@gmail.com
}

\begin{abstract}
$D E \backslash B B O$ is the hybridization of Differential Evolution optimization and Biogeography Based Optimization. DE is considered to have good exploration ability and BBO is considered to have good exploitation ability, to achieve goodness of both techniques, these techniques have been combined. Hybridization of Differential evaluation and biogeography based optimization dominates the performance of biogeography based optimization. The influencing factors of performance of DE are scaling factor and crossover rate which are used to track proper optimization. In this paper, it has been shown how $D E / B B O$ outperforms $B B O$. The change in the performance of $D E / B B O$ due to variation in some pre-considered variables has also been highlighted. It has been observed that $D E / B B O$ performs best when scaling factor is 0.11 and cross over rate is 0.2 .
\end{abstract}

Keywords: Differential evaluation, Biogeography based optimization, mutation, migration

\section{Introduction}

Differential Evolution [1] was proposed to index the global optimization. Before the introduction of Differential Evolution, various other optimization methods were used which did not include any objective function. DE made use of objective function to address the global optimization. In this, mutation, crossover and selection procedures are used where mutation is used to generate the new vector, crossover is used to pick the member of population that needs modification and selection is used to decide whether to include the modified member into population or not. Mutation refers to selection of three random members from population and generation of new vector as below:

New vector $=$ First member + Scaling factor* $($ Second member - Third member $)$

Biogeography based optimization [5] was proposed by Dan Simon whose inspiration came from biological distribution of species in different geographical areas. Mathematical model of biogeography has been taken from the theory of island biogeography. These models show the behavior of organisms under various conditions. If the environment is suitable for living, then organisms will migrate to that particular environment otherwise they will emigrate to other environment or we can say other habitat or island. HSI (Habitat suitability index) determines the suitability of the habitat for being on that habitat. SIV (Suitability Index variables) characterize the habitability of particular habitat. The same concept can be applied to real life problems in any area like engineering, economics, medicine etc. If there is a problem then its solutions are considered as habitats. Solutions with high value of HSI are considered as good solutions whereas solutions with low value of HSI are considered as bad solutions. Solutions with high HSI do not accept features of other solutions so as to maintain its own goodness but solutions with low HSI accept the features of good solutions and are improved generation to generation. Immigration rate and emigration rate decides which solution will take features 
of other solution and which will give features to other solutions respectively. If number of species is low, then immigration rate is higher otherwise emigration rate is higher. Migration is done to share information between the various solutions. Note that HSI decides the goodness of a solution but SIV are integers of a vector which determines the population of each candidate solution. In migration, firstly SIV (that is to be modified) of a particular solution is selected on basis of immigration rate. Then, emigration rate of other solutions decide that which solution will give randomly selected SIV value to the selected solution which is to be modified. Hence bad solutions are modified. Mutation is done to maintain the solution's goodness to a particular level. According to probability, each solution is modified so that none of solution becomes the worse solution.

$\mathrm{DE} / \mathrm{BBO}[8]$ concentrates on improving the searching capability of differential algorithm by using effectiveness of BBO. It is the combination of goodness of both algorithms that is mutation, crossover, selection operator of differential algorithm and migration operator of Biogeography based optimization which in turn generates very promising solutions. DE is famous for yielding faster and better solutions but problem occurs when the complexity of the problem, that is to be solved, increases. It is due to presence of crossover rate which tend to decrease the quality of solution at the end. There is no such problem in BBO. The proposed algorithm gives the better quality solutions while providing better robustness and convergence characteristics as compared to original BBO. Steps illustrating the hybridization of Differential Evolution and Biogeography Based Optimization are as given below:

Step 1: Initialization of population, other parameters of BBO such as immigration rate, emigration rate, Mutation probability etc. and calculation of cost of each member of population.

Step 2: Modify the population using hybrid migration operator.

Step 3: Calculate the cost of modified population with previous generation population. Keep the better.

Step 4: Repeat Step 2 and Step 3 till the last generation.

Remaining paper has been organized in various sections. Literature survey has been given in Section 2. Section 3 illustrates the concept of hybridization of DE and BBO. The results taken by running DE/BBO in MATLAB environment has been shown in section 4 and 5. Then the paper has been concluded with reflection of future work in section 6 .

\section{Related Work}

Biogeography based optimization method [5] has been given by Dan Simon in 2008. This method has its roots in the natural way of distribution of species in geographical area. The idea of species migration from one habitat to another on the basis of suitability of that particular habitat has been utilized to improve the quality of solutions so as to get optimum solution. Original BBO has many deficiencies. To improve original BBO, many other versions of BBO have been introduced. In 2009, Oppositional BBO [6] has been introduced which focuses on improving the convergence speed by using only migration not mutation. In 2010, real coded BBO [7] has been introduced in which modified mutation operators are used to improve the performance of original BBO. In 2010, $\mathrm{DE} / \mathrm{BBO}[8]$ has been introduced which provides better robustness and increased convergence speed. In 2012, Blended BBO [9] has been introduced which includes new way of changing the features of solution. In 2012, Multi-objective BBO [10] based on predator-prey approach has been introduced which focuses on increasing the capability of finding good solutions. In 2014, Hybrid BBO [11] has been introduced which includes the combination of various evolutionary algorithms with BBO. In 2014, Linearized BBO [12] has been introduced for highly non-separable problems.

Differential Evaluation optimization method [1] has been given by Storn R and Price $\mathrm{K}$ in 1995. In this, trial vector is generated from three vectors randomly 
selected from the population. Then crossover and selection are applied. The limitation of DE is premature convergence which has been improved by changing its mutation operator and introducing modified crossover processes. Various modified mutation schemes are DElbest $\backslash 1$, DEltarget-to-bestlt, DE $\backslash$ best $\backslash 2$, DE $\backslash$ rand $\backslash 2$ etc. Recent work on modification in mutation scheme has been found in 2012 and 2014 which focus on local search mutation [13]. This results in improvement of the performance of $\mathrm{DE}$ in various means like efficiency, quality of solution and robustness.

\section{Concept of Hybridization of DE\BBO}

Differential evaluation is considered to have good exploration ability which means searching of new individuals from the current individuals but when the problem size increases, the efficiency of DE decreases. The solutions which are good at initial step may get worst after the final step. To avoid this problem, DE mutation has been combined with migration of $\mathrm{BBO}$ which is known as hybrid $\mathrm{DE} / \mathrm{BBO}$ approach [8] for optimization process. In the algorithm, $\mathrm{F}$ and $\mathrm{CR}$ the pre-considered variables known as scaling factor and cross-over rate respectively, value of $\mathrm{F}$ can vary from 0 to 2 and CR can vary from 0 to 1 .

Algorithm describing the process of hybrid migration of $\mathrm{DE} / \mathrm{BBO}[8]$ is as given below:

a. for $\mathrm{G}=1$ to $\mathrm{GL} / / \mathrm{GL}$ is generation limit.

b. for $\mathrm{n}=1$ to $\mathrm{PS} / / \mathrm{PS}$ is population size.

c. Randomly select three members so that $\mathrm{a}_{1 \neq} \mathrm{a}_{2 \neq} \mathrm{a}_{3 \neq} \mathrm{n}$

d. $\quad \mathrm{q}_{\mathrm{rand}}=\operatorname{randi}(1, \mathrm{~m}) / /$ randi is used to choose random integer from given numbers.

e. for $\mathrm{q}=1$ to $\mathrm{m} / / \mathrm{m}$ is population dimension.

f. if rand $<\lambda_{\mathrm{n}} \quad / / \lambda$ is immigration rate.

g. if $\operatorname{rand}_{\mathrm{q}}>\mathrm{CR}$ or $\mathrm{q}==\mathrm{q}_{\mathrm{rand}} \quad / / \mathrm{CR}$ is crossover rate.

h. $\quad J_{n}(q)=X_{a 1}(q)+F\left(X_{a 2}(q)-X_{a 3}(q)\right) / / F$ is scaling factor.

i. else

j. $\quad$ Randomly select $X_{\mathrm{h}}$ with probability $\mu_{\mathrm{h}}$

k. $\quad \mathrm{J}_{\mathrm{n}}(\mathrm{q})=\mathrm{X}_{\mathrm{h}}(\mathrm{q})$

1. end

m. else

n. $\quad \mathrm{J}_{\mathrm{n}}(\mathrm{q})=\mathrm{X}_{\mathrm{n}}(\mathrm{q})$

o. end

p. end

q. end

r. end

In the above algorithm, $\mathrm{CR}$ and $\mathrm{F}$ are the constants used in differential evaluation optimization where $\mathrm{CR}$ is crossover rate varying from 0 to 1 and $\mathrm{F}$ is scaling factor varying from 0 to 2 .

This algorithm describes the migration process which has been combined using the mutation of differential evaluation and migration of biogeography based optimization. 


\section{Fluctuation in Results by Varying $F$ and $C R$ values}

The performance of $\mathrm{DE} / \mathrm{BBO}$ has been studied by varying the scaling factor $\mathrm{F}$ from 0 to 2 and crossover factor CR from 0 to 1 on MATLAB software R2012b, 32 bit window 7 and RAM 3.00 GB. Scaling factor F is considered to be $0.2,0.5,0.8,0.11,0.14,0.17,2$ and Crossover rate is considered to be $0.2,0.4,0.6,0.8$, and 1 . The variation in the cost, percentage of minimization of cost and convergence speed examined by taking different values of $\mathrm{F}$ and $\mathrm{CR}$ has been pin pointed in Table 1, Table 2 and Table 3 respectively.

Running time of $\mathrm{BBO}$ is approximately 3.131 seconds. As observed from Table 3, CPU time for DE $\backslash B B O$ is less than $\mathrm{BBO}$ for $\mathrm{F}$ from 0.2 to 0.14 and $\mathrm{CR}=0.2,0.4$. But when looking at the percentage of minimization, only few combinations of $F$ and $C R$ outperform BBO (Minimization percentage 97.12 approx.). These combinations are $\mathrm{F}=$ 0.11 and $\mathrm{CR}=0.2, \mathrm{~F}=0.2$ and $\mathrm{CR}=0.4, \mathrm{~F}=0.11$ and $\mathrm{CR}=0.4$. If both running time and percentage of minimization is to be taken into account then suitable combination is $\mathrm{F}=$ 0.11 and $\mathrm{CR}=0.2$.

Figure 1 shows convergence of $\mathrm{DE} \backslash \mathrm{BBO}$ algorithm at $\mathrm{F}=0.2$ and $\mathrm{CR}=0.2,0.4$. In the same way, rest of Figurers from Figure 2 to Figure 6 shows convergence characteristics of DE $\backslash B B O$ for various combinations of $F$ and $C R$.

Table 1. Maximum Cost and Minimum Cost For Different Values of F and CR

\begin{tabular}{|c|c|c|c|c|c|c|}
\hline $\mathrm{CR}$ & & 0.2 & 0.4 & 0.6 & 0.8 & 1 \\
\hline \multirow[t]{2}{*}{0.2} & $\begin{array}{l}\text { Max } \\
\text { Cost }\end{array}$ & 2210.7483 & 2803.4806 & 4236.7899 & 4050.3128 & 4304.2406 \\
\hline & $\begin{array}{c}\text { Min } \\
\text { Cost }\end{array}$ & 144.5644 & 71.0857 & 78.9915 & 59.7302 & 128.7639 \\
\hline \multirow[t]{2}{*}{0.5} & $\begin{array}{c}\text { Max } \\
\text { Cost }\end{array}$ & 3363.5122 & 2963.5488 & 4070.6872 & 4094.676 & 3600.1896 \\
\hline & $\begin{array}{l}\text { Min } \\
\text { Cost }\end{array}$ & 959.1732 & 798.2002 & 151.2922 & 103.8729 & 132.211 \\
\hline \multirow[t]{2}{*}{0.8} & $\begin{array}{l}\text { Max } \\
\text { Cost }\end{array}$ & 3438.8241 & 3743.2242 & 3474.1933 & 2706.4322 & 3133.6853 \\
\hline & $\begin{array}{c}\text { Min } \\
\text { Cost }\end{array}$ & 3173.241 & 1448.7841 & 798.9554 & 247.2559 & 130.3291 \\
\hline \multirow[t]{2}{*}{0.11} & $\begin{array}{l}\text { Max } \\
\text { Cost }\end{array}$ & 4583.9421 & 3546.9031 & 4450.7566 & 3489.6403 & 5196.9771 \\
\hline & $\begin{array}{c}\text { Min } \\
\text { Cost }\end{array}$ & 111.9542 & 80.9988 & 78.7398 & 62.3844 & 187.6929 \\
\hline \multirow[t]{2}{*}{0.14} & $\begin{array}{l}\text { Max } \\
\text { Cost }\end{array}$ & 4266.4411 & 4169.8674 & 4477.6703 & 4513.2741 & 4569.1875 \\
\hline & $\begin{array}{c}\text { Min } \\
\text { Cost }\end{array}$ & 187.774 & 122.4853 & 138.6704 & 111.025 & 153.5529 \\
\hline \multirow[t]{2}{*}{0.17} & $\begin{array}{c}\text { Max } \\
\text { Cost }\end{array}$ & 3004.1959 & 3259.9541 & 3094.2452 & 3867.0626 & 3072.932 \\
\hline & $\begin{array}{c}\text { Min } \\
\text { Cost }\end{array}$ & 151.7914 & 131.5858 & 100.0615 & 87.9332 & 143.0761 \\
\hline \multirow[t]{2}{*}{2} & $\begin{array}{c}\text { Max } \\
\text { Cost }\end{array}$ & 2597.2421 & 4097.5729 & 2666.0819 & 2799.9188 & 2862.6699 \\
\hline & $\begin{array}{c}\text { Min } \\
\text { Cost }\end{array}$ & 5860.831 & 3385.9158 & 4414.2317 & 1963.0557 & 164.2672 \\
\hline
\end{tabular}


Table 2. CPU time Utilized in Seconds for Different Values of $F$ and CR

\begin{tabular}{|c|c|c|c|c|c|}
\hline \multicolumn{1}{|c|}{$\begin{array}{c}\mathrm{CR} \\
\mathrm{F}\end{array}$} & 0.2 & 0.4 & 0.6 & 0.8 & 1 \\
\hline 0.2 & & & & & \\
\hline 0.5 & 1.648 & 2.744 & 3.522 & 4.266 & 5.32 \\
\hline 0.8 & 1.67 & 2.722 & 3.55 & 4.466 & 5.304 \\
\hline 0.11 & 1.79 & 2.54 & 3.536 & 4.427 & 5.203 \\
\hline 0.14 & 1.661 & 2.63 & 3.368 & 4.254 & 5.309 \\
\hline 0.17 & 1.714 & 2.614 & 8.469 & 10.701 & 13.033 \\
\hline 2 & 3.989 & 6.481 & 8.358 & 9.957 & 12.747 \\
\hline
\end{tabular}

Table 3. Percentage of Minimization

\begin{tabular}{|c|c|c|c|c|c|}
\hline & 0.2 & 0.4 & 0.6 & 0.8 & 1 \\
\hline 0.2 & 93.4608 & 97.4644 & 98.1356 & 98.5253 & 97.0084 \\
\hline 0.5 & 71.4829 & 73.0661 & 96.2833 & 97.4632 & 96.3277 \\
\hline 0.8 & 7.7231 & 61.2958 & 77.0031 & 90.8641 & 95.841 \\
\hline 0.11 & 97.5576 & 97.7164 & 98.2309 & 98.2123 & 96.3884 \\
\hline 0.14 & 95.5988 & 97.0626 & 96.9031 & 97.54 & 96.6394 \\
\hline 0.17 & 94.9473 & 95.9636 & 96.7662 & 97.7260 & 95.3439 \\
\hline 2 & $\begin{array}{c}\text { Cost does } \\
\text { not minimized }\end{array}$ & 17.3678 & $\begin{array}{c}\text { Cost does } \\
\text { not minimized }\end{array}$ & 29.8888 & 94.2617 \\
\hline
\end{tabular}

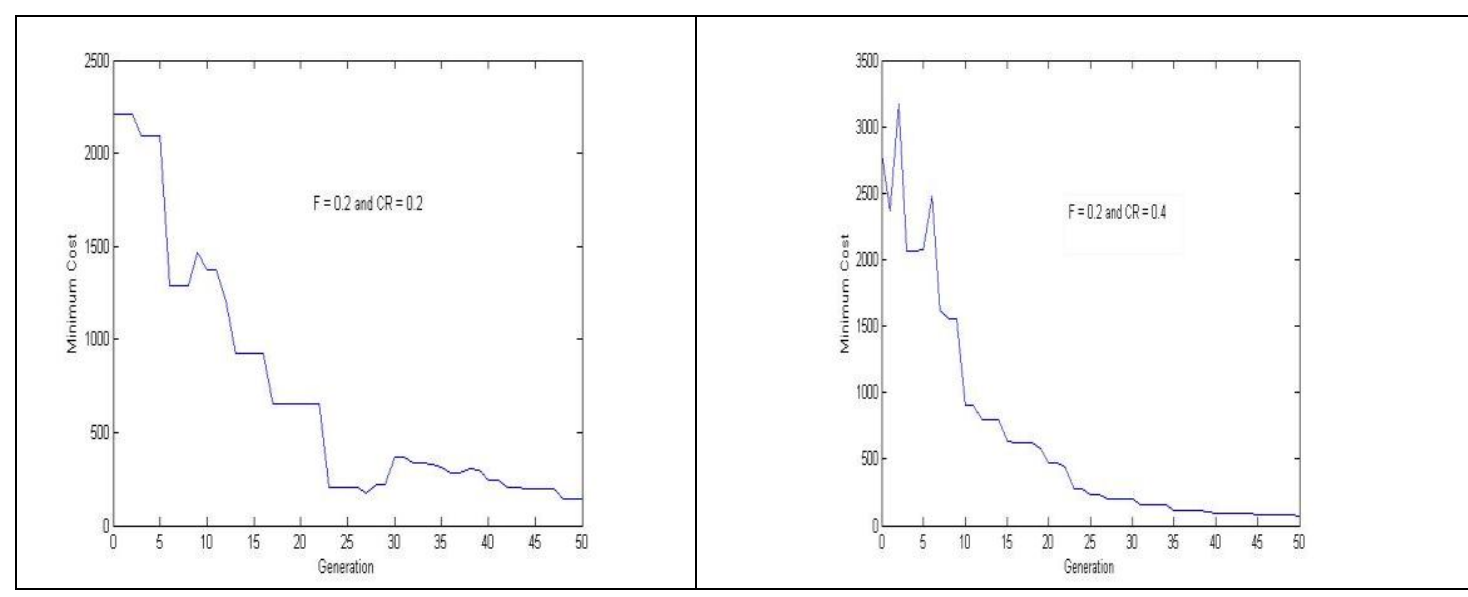

Figure 1. Graph Showing Minimum Cost For Each Generation for F = 0.2 and $\mathrm{CR}=0.2,0.4$

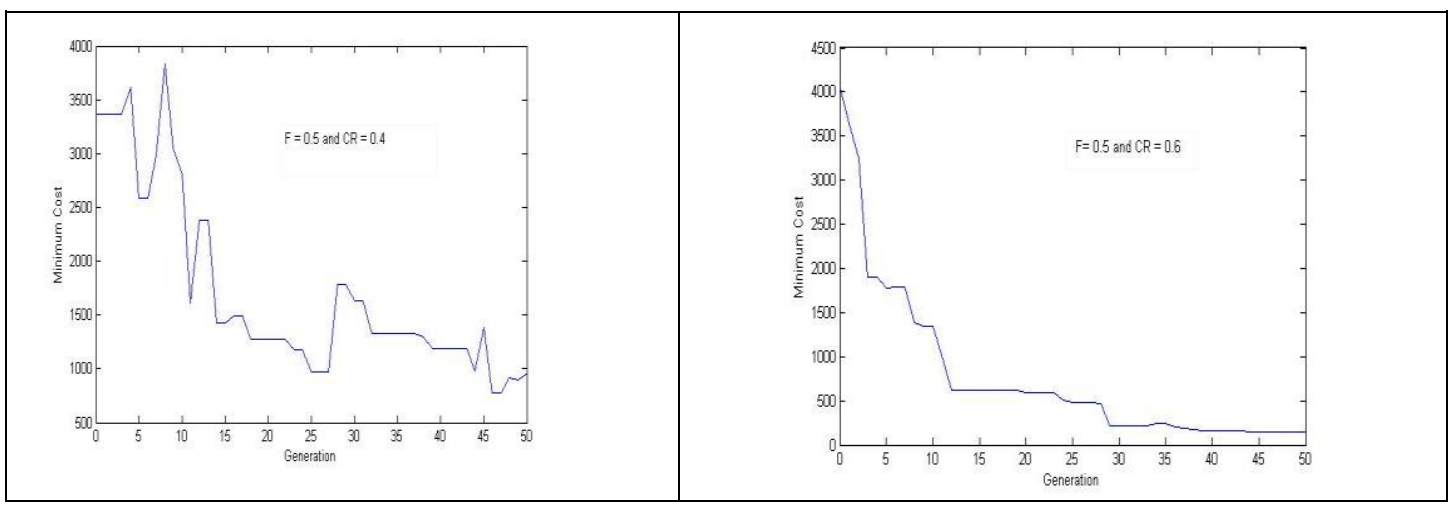


Figure 2. Graph Showing Minimum Cost For Each Generation for $F=0.5$ and $\mathrm{CR}=0.2,0.6$

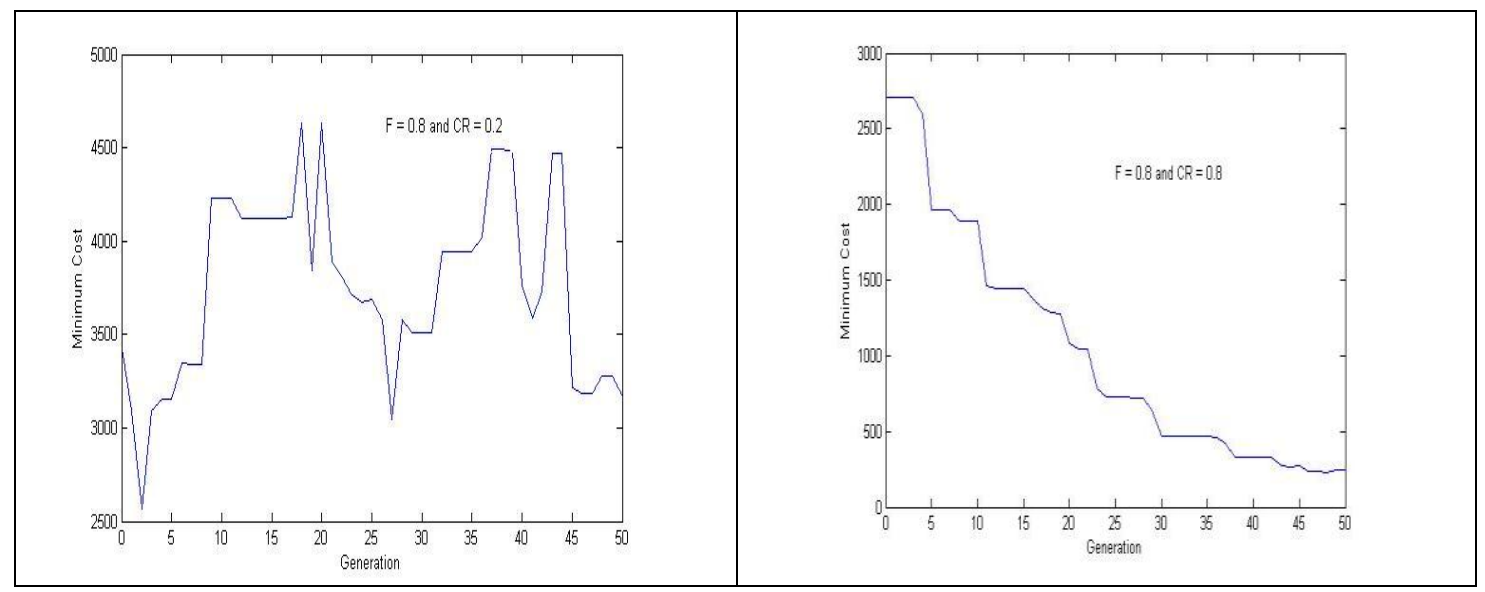

Figure 3. Graph Showing Minimum Cost For Each Generation for $F=0.8$ and $\mathrm{CR}=0.2,0.8$

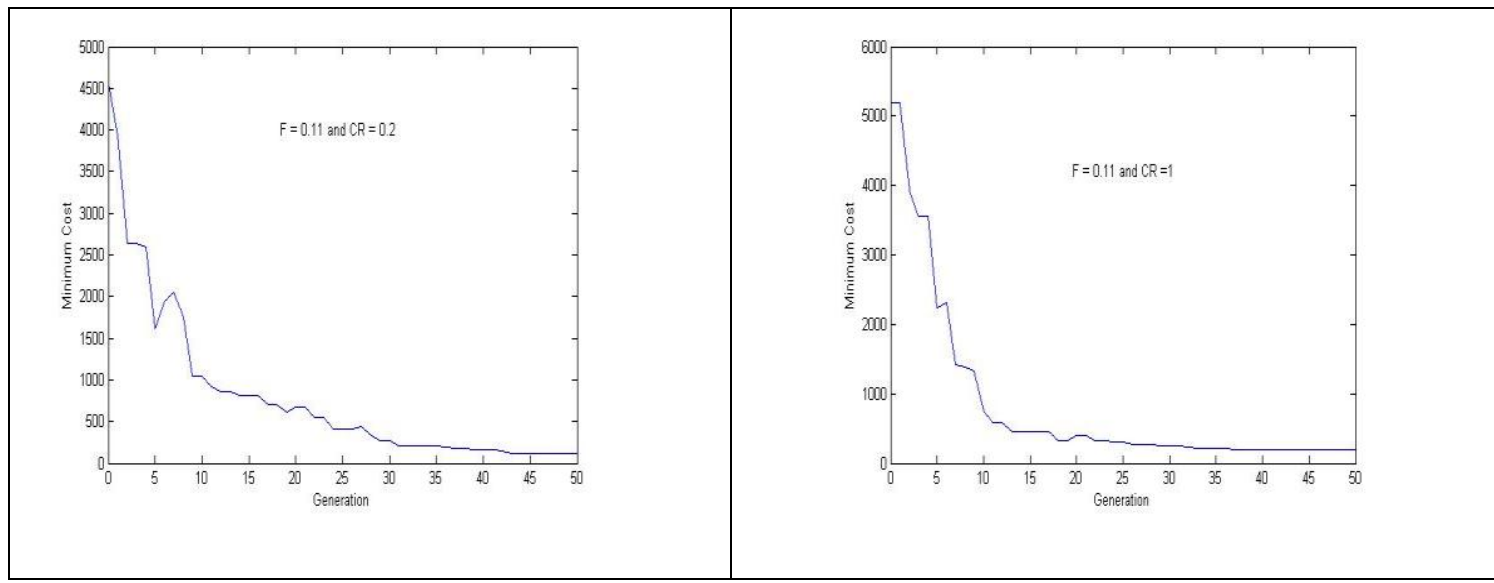

Figure 4. Graph Showing Minimum Cost For Each Generation for F = 0.11 and $C R=0.2,1$

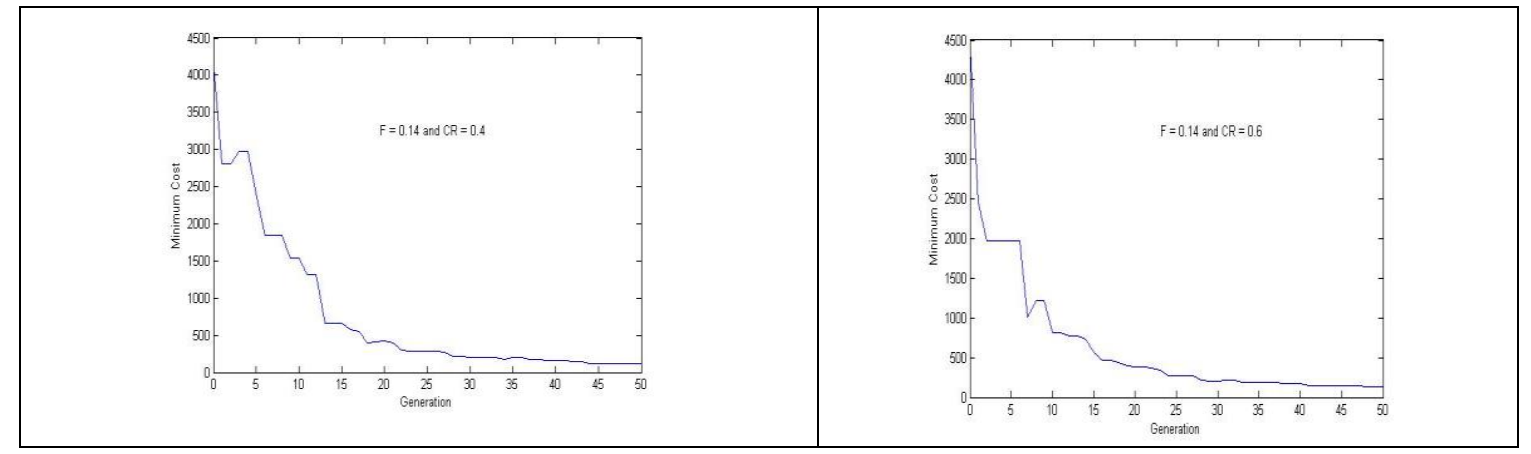

Figure 5. Graph Showing Minimum Cost For Each Generation for F = 0.14 and $\mathrm{CR}=0.4,0.6$ 


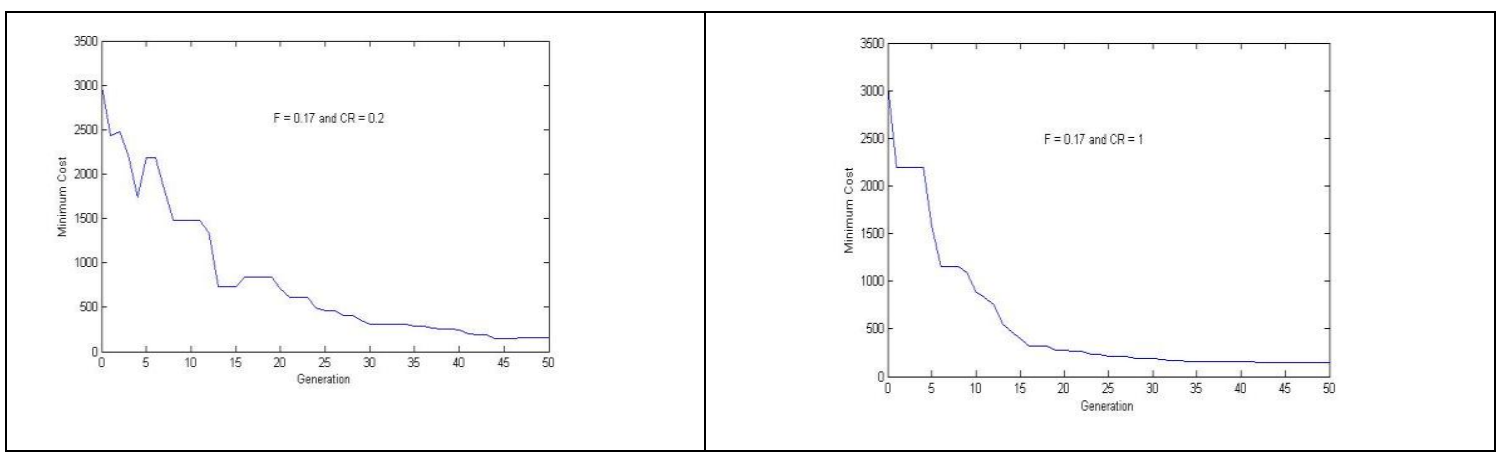

Figure 6. Graph Showing Minimum Cost For Each Generation for F $=0.17$ and $\mathrm{CR}=0.2,1$

\section{Comparative Analysis of BBO and DE\BBO}

When performance of $\mathrm{BBO}$ is compared with $\mathrm{DE} \backslash \mathrm{BBO}$, it has been observed that DE\BBO performs better than BBO giving improvement in convergence speed. Figure 7 illustrates the performance of both algorithms.

\begin{tabular}{|c|c|c|c|c|c|}
\hline \multicolumn{8}{|c|}{$\mathrm{BBO}$ v/s DE/BBO( $\mathrm{F}=0.11$ and $\mathrm{CR}=0.2)$} \\
\hline BBO with Convergence speed $=3.131 \mathrm{~s}$ & $\mathrm{DE} / \mathrm{BBO}$ with convergence speed $=1.661 \mathrm{~s}$ \\
\hline
\end{tabular}

Figure 7. Graph Showing Convergence for BBO and DE\BBO

\section{Conclusion and Future Scope}

This paper is dedicated to evaluate the performance of DE $\backslash \mathrm{BBO}$ by varying scaling factor and cross over rate. From the performance evaluation, it can be concluded that BBO takes more time to converge than DE\BBO. Performance of DE $\backslash B B O$ fluctuates very rapidly with increase in crossover rate. For scaling factor $F$ $=0.2$, convergence speeds are $1.648,2.744,3.522,4.266,5.32$ seconds for corresponding crossover rate $0.2,0.4,0.6,0.8$ and 1 respectively. Figure 8 conforms to the conclusion of increase in convergence speed with increase in crossover rate.

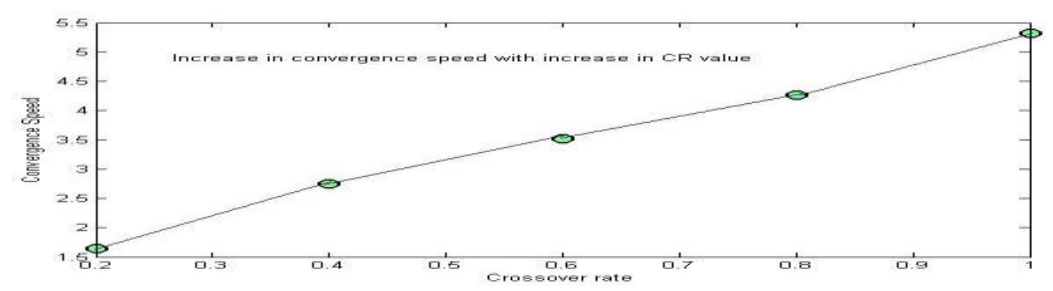




\section{Figure 8. Illustration of Increase in Convergence Speed with Increase in Crossover Rate for $\mathbf{F}=\mathbf{0 . 2}$}

DE $\backslash B B O$ evaluated in this paper is based on simple mutation technique. But in the survey of differential evolution optimization, it has been noticed that there are many new mutation mechanism. In future, DE\BBO can be modified by using the recent mutation mechanism and corresponding performance can be evaluated.

\section{References}

[1] R. Storn and K. V. Price, "Differential evolution- A simple and efficient adaptive scheme for global optimization over continuous spaces", Technical Report TR-95-012, ICSI, (1995).

[2] R. Storn and K. V. Price, "Differential Evolution- a simple and efficient heuristic for global optimization over continuos spaces”, Journal of Global Optimization, vol. 11, no. 4, pp. 341-359, (1997).

[3] R. Storn, K. V. Price and J. Lampinen, "Differential Evolution- A Practical Approach to Global Optimization", Springer, Berlin, (2005).

[4] D. Swagatam, A. Ajith and K. Uday, "Differential Evolution Using a Neighborhood-based Mutation Operator", (2007).

[5] D. Simon, "Biogeography-based optimization", IEEE Transactions on Evolutionary Computation, vol. 12, no. 6, (2008) December, pp. 702-713.

[6] M. Ergezer, D. Simon and D. Du, "Oppositional Biogeography-Based Optimization", Proceedings of the 2009 IEEE International Conference on Systems, Man, and Cybernetics San Antonio, TX, USA October (2009).

[7] W. Gong, Z. Cai, C. X. Ling and H. Li, "A real coded Biogeography based optimization with mutation", Applied Mathematics and Computation, vol. 216, (2010), pp. 2749-2758.

[8] A. Bhattacharya, Member, IEEE and P. K. Chattopadhyay, "Hybrid Differential Evolution With Biogeography-Based Optimization for Solution of Economic Load Dispatch", IEEE transactions on power systems, vol. 25, no. 4, (2010) November.

[9] S. Kanoongo and P. Jain, "Blended Biogeography Based Optimization For Different Economic Load Dispatch Problem", IEEE Canadian Conference on Electrical and Computer Engineering (CCECE), (2012).

[10] M. A. C. e Silva, L. dos S. Coelho and L. Lebensztajn, "Multi objective Biogeography- Based Optimization Based on predator-Prey Approach", IEEE Transactions On Magnetics, vol. 48, no. 2, (2012) February.

[11] D. Simon, G. H. M. Omran and M. Clerc, "Linearized Biogeography-Based Optimization with Reinitialization and Local search", Information Sciences, vol. 267, (2014), pp. 140-157.

[12] M. Haiping, D. Simon, M. Fei, X. Shu and Z. Chen, "Hybrid Biogeography-Based evolutionary algorithms", Engineering Applications of Artificial Intelligence, vol. 30, (2014), pp. 213-224.

[13] A. W. Mohamed, "RDEL, Restart Differential Evolution algorithm with Local Search Mutation for global numeric optimization”, Egyptian Informatics Journal, (2014). 